\title{
ARTICLE
}

\section{Gross pathology of herons and egrets (family Ardeidae) at a wildlife rehabilitation centre in Northern California}

\author{
Molly Horgan ${ }^{1} \&$ Rebecca Duerr ${ }^{2}$ \\ 'School of Veterinary Medicine, University of California-Davis, Davis, CA, USA; \\ ${ }^{2}$ International Bird Rescue, Fairfield, CA, USA
}

\begin{abstract}
Gross necropsies were performed on all herons and egrets (family Ardeidae) that died or were euthanized at a wildlife rehabilitation centre in Fairfield, California, over a six-week period in the summer of $2018(n=145)$. Of the animals necropsied, $108(74.5 \%)$ were euthanized and 37 (25.5\%) died. Common reasons for euthanasia were trauma $(n=24), \operatorname{MBD}(n=39)$ and non-specific signs such as hypothermia and obtundation that persisted in the face of medical and supportive care $(n=32)$. MBD was found both in birds at admission $(n=9)$ and later in care $(n=34)$, despite calcium supplementation to correct calcium:phosphorus ratios of foods. Feeder fish were suspected to be deficient in vitamin D, and supplementation successfully reduced the development of this problem during the care later in the season. Nematodes of the genus Eustrongylides were found in the coelomic cavities of $43(30 \%)$ birds. High burdens were often accompanied by coelomitis and abscessation involving internal organs. Visceral gout $(n=10)$ and intestinal perforation $(n=4)$ were both strongly associated with the administration of meloxicam. These findings suggest meloxicam should be used with caution in these species. Other less common findings included septic arthritis, intestinal perforation and hepatomegaly. This study describes common gross pathological findings of herons and egrets in a wildlife rehabilitation centre in California and demonstrates the utility of gross necropsies in determining cause of death when more advanced post-mortem diagnostics are unavailable.
\end{abstract}

BIO

Molly Horgan obtained her DVM from the University of California, Davis, in 2020. She is currently completing a residency in exotic, wildlife, and zoological medicine at the University of Florida. Before enrolling in veterinary school, she completed three internships at wildlife rehabilitation centers (and volunteered in several others) and hopes to continue working in the field of wildlife medicine and conservation. She is also interested in research pertaining to wildlife medicine and disease.

Rebecca Duerr is the clinical veterinarian and research director at International Bird Rescue's two wildlife clinics in California. She completed her DVM, MPVM and PhD degrees at the UC Davis, with a graduate work on the care of oiled Common Murres and on the nutritional energetics and physiology of Common Murres and Western Grebes. She co-edited the second edition of Hand-Rearing Birds and Topics in Wildlife Medicine IV: Orthopedics and co-wrote the wild orphans section of Merck Veterinary Manual.

\section{Introduction}

High patient loads in wildlife rehabilitation centres, especially during the spring and summer, mean that it is often not feasible to perform necropsies due to lack of time and resources. Because of this, the cause of death of animals that die suddenly in care, or are euthanized for declining clinically, often goes unknown. Although

\section{Keywords}

Ardeids; egret; Eustrongylides; gout; heron; meloxicam; metabolic bone disease; NSAID; necropsy; pathology; renal failure; vitamin D; wildlife rehabilitation

\section{Correspondence}

Molly Horgan, DVM. University of Florida College of Veterinary Medicine. 2089 SW 16th Avenue, Gainesville FL 32609

Email: horganm@ufl.edu

\section{Abbreviations}

BCNH: black-crowned night herons BID: twice daily, q12h

CAEG: cattle egrets

COX: Cyclooxygenase, an enzyme important in the metabolic pathways involving inflammation

GBHE: great blue herons

GREG: great egrets

GRHE: green herons

MBD: metabolic bone disease

NSAID, non-steroidal anti-inflammatory drug

SID: once daily, q24h

SNEG: snowy egrets

Dates

Accepted: 23 April 2020

Published: 29 November 2021 
are frequently admitted to wildlife rehabilitation centres. Published reports on the pathology of herons and egrets are scarce, but include studies on haemoparasites of ardeids at a wildlife rehabilitation centre in Florida (Coker et al. 2017), erythrocyte nuclear abnormalities in grey herons (Ardea cinerea) at a wildlife rehabilitation centre in Spain (Santos et al. 2017), MBD in free-ranging CAEG (Bulbulcus ibis) in Texas (Phalen et al. 2005) and in grey herons in the UK (Thompson et al. 2006) and eustrongylidiasis, an important parasitic cause of mortality in young herons and egrets, in multiple areas of the United States, including California (Wiese et al. 1977; Spalding \& Forrester 1993; Spalding et al. 1993; Franson \& Custer 1994; Ziegler et al. 2000).

The purpose of this study was to characterize gross pathological findings in herons and egrets at a wildlife rehabilitation centre and attempt to determine the cause of death or clinical signs leading to euthanasia in animals where it was not obvious pre-mortem.

Table 1 Number of animals necropsied by species and life stage.

\begin{tabular}{lc}
\hline Species & Number \\
\hline Black-Crowned Night Heron (Nycticorax nycticorax) & 33 \\
Cattle Egret (Bulbulcus ibis) & 9 \\
Great Blue Heron (Ardea Herodias) & 2 \\
Great Egret (Ardea alba) & 7 \\
Green Heron (Butoroides virescens) & 21 \\
Snowy Egret (Egretta thula) & 73 \\
Life stage & \\
Hatchling & 5 \\
Nestling & 35 \\
Fledgling & 95 \\
Older juvenile & 9 \\
Adult & 1 \\
\hline
\end{tabular}

\section{Materials and methods}

\section{Necropsies}

Six species of heron and egret (family Ardeidae) were included in the study: SNEG (Egretta thula) $(n=73)$, BCNH (Nycticorax nycticorax) $(n=33)$, GRHE (Butoroides virescens) $(n=21)$, CAEG (Bulbulcus ibis) $(n=9)$, GREG (Ardea alba) $(n=7)$ and GBHE (Ardea herodias) $(n=2)$ (Table 1). Gross necropsies were performed on all included species that died or were euthanized during a six-week period from mid-June to early August 2018. Necropsies were performed within three days of death, and carcasses were kept refrigerated at $4{ }^{\circ} \mathrm{C}$ between death and necropsy. Body condition scoring was performed as described by van Franeker (2004). The diameter of parathyroid glands was measured at the widest point with a ruler to the nearest millimetre. Longitudinal cross-sectioning of one tarsometatarsus of each bird was attempted with a \#10 scalpel blade starting at the proximal epiphysis and proceeding distally. The ease of cross sectioning was recorded. Birds were defined as having MBD at necropsy when they had folding fractures or angular deformities of long bones and/or bones that were easily cut with a \#10 scalpel blade. All necropsies were performed by the same author $(\mathrm{MH})$. Gross lesions were digitally photographed.

\section{Records}

Medical records for each animal were searched to determine the reason(s) for euthanasia, clinical signs preceding death and any history of drug administration for each bird necropsied. A summary of the number of animals that died and were euthanized is given in Fig. 1 . To better assess the incidence of MBD in 2018 and how

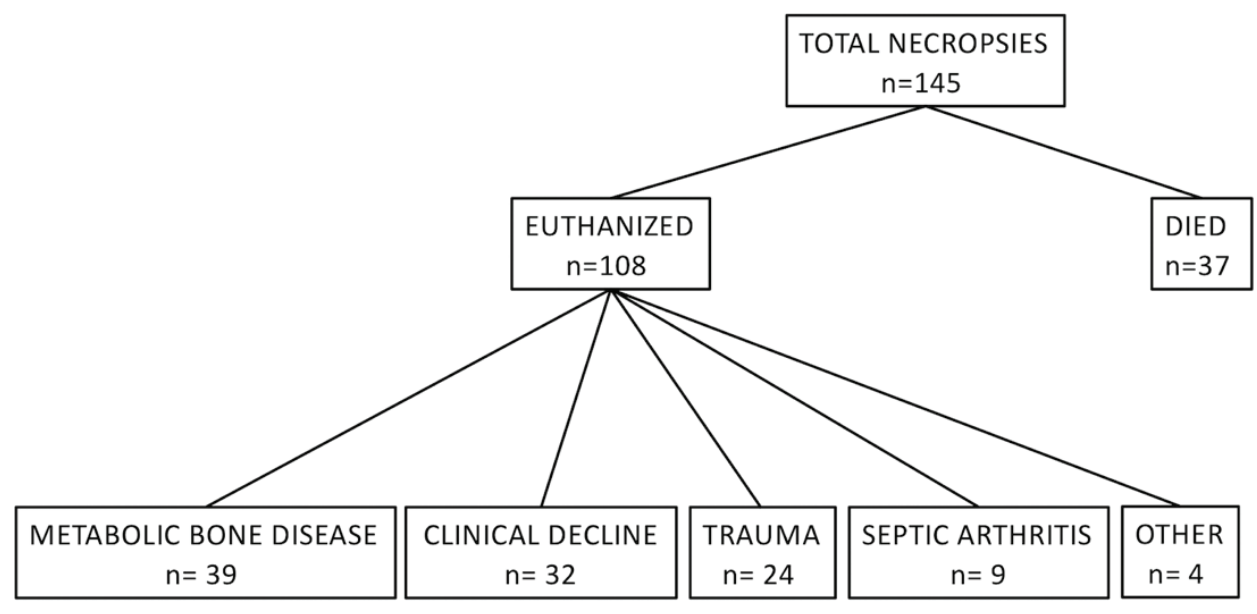

Fig. 1 Number of animals included in this study that died and were euthanized, and the reasons for euthanasia as stated in medical records. 
it compared to the prior year, the records of all herons and egrets that had intake dates between 1 April and 9 August of both 2017 and 2018 were searched. Birds were defined as having MBD if their records noted boney angular deformities, folding fractures, soft bones on palpation or a stated diagnosis of MBD. Cases were defined as having MBD on intake if characteristics of MBD were present at the intake exam or within three days of admission. Cases were defined as having acquired MBD in care if MBD was noted four days or later after intake. Birds were defined as being euthanized for clinical decline if their record noted weight loss, persistent hypothermia, persistent obtundation and/or agonal breathing as the cause of euthanasia.

\section{Cytology}

The Diff-Quik stain (Baxter Diagnostics, Deerfield, IL) was used to stain samples of joint effusion for cytology. Samples were taken from the affected joints of two birds euthanized for presumed septic arthritis of the elbow and hock.

\section{Statistics}

Parathyroid gland diameter of birds with and without a clinical MBD was compared using T-test. The associations between meloxicam treatment and visceral gout, and meloxicam treatment and intestinal perforation were analysed using Fisher's exact tests with $2 \times 2$ contingency tables. Significance was set at $p=0.05$.

\section{Results}

The three most common species necropsied were SNEG $(n=73), \mathrm{BCNH}(n=33)$ and GRHE $(n=21)$, and the majority of animals were either nestlings $(n=35)$ or fledglings $(n=95)$ (Table 1$)$. Common pathological findings included $\operatorname{MBD}(n=47)$, eustrongylidiasis $(n=43)$, liver abscesses $(n=14)$, visceral gout $(n=10)$ and septic arthritis $(n=9)$ (Table 2$)$.

Table 2 Total numbers of animals with major gross pathological findings.

\begin{tabular}{lc}
\hline Finding & Number of animals affected \\
\hline Metabolic bone disease & 47 \\
Eustrongylidiasis & 43 \\
Liver abscess(es) & 14 \\
Visceral gout & 10 \\
Septic arthritis & 9 \\
Lung abscess(es) & 8 \\
Intestinal perforation & 4 \\
\hline
\end{tabular}

\section{Metabolic bone disease}

MBD findings are fully reported in a separate paper (Horgan et al. 2021). Briefly, MBD was the leading cause of euthanasia in the period of time during which necropsies were conducted. Of the animals necropsied, 43 had signs of MBD, 39 of which were euthanized due to MBD. According to records, nine of the necropsied birds had signs of MBD on intake, and 34 developed signs after four or more days in care. Clinically, MBD was characterized by folding fractures and angular deformities of long bones, most commonly the tarsometatarsus, as well as the increased flexibility of the maxilla and long bones. On necropsy, affected long bones were easy to cut through with a \#10 scalpel blade, particularly at the physes. Cross sections of the tarsometatarsus revealed fractures and long, grossly irregular, gelatinous-appearing physeal zones (Fig. 2). The average diameter of the parathyroid glands of affected birds was significantly larger than those of birds unaffected by MBD $(p=0.0001)$. The parathyroid gland releases parathyroid hormone in response to hypocalcemia, so gland hypertrophy can be seen in MBD.

Vitamin D deficiency due to low levels in the birds' diet of capelin (Mallotus villosus) was proposed as the possible cause for the high number of MBD cases developing in care, as the chicks were already receiving calcium supplementation to correct for a known dietary deficiency. At the end of the third week of the study, the centre began supplementing oral vitamin $\mathrm{D}_{3}$ at $714 \mathrm{IU} / \mathrm{kg} /$ day, after which the number of animals developing MBD in care dropped dramatically (Fig. 3). Birds with signs of MBD that were necropsied after the vitamin D supplementation began $(n=7)$ had intake dates at least two weeks before the supplementation began.

A search of records of all herons and egrets with intake dates from 1 April to 9 August during 2017 and 2018 showed a substantial increase in birds with MBD at intake in 2018 compared to 2017, an increase largely attributable to a higher number of cases from Santa Rosa (Table 3). No diagnostics were performed to determine the reason for the increase in MBD cases at admission in 2018 compared to 2017.

\section{Endoparasites}

White nematode worms of $1-3 \mathrm{~cm}$ in length were present within the proventriculus and/or ventriculus of 11/145 $(7.6 \%)$ of animals. They were not identified to species. All affected birds had died or had been euthanized within four days of admission. Flukes, presumed to be Clinostomum marginatum, were found in the mouth and/or cranial oesophagus of 5/145 (3.4\%) of birds. Avioserpens species worms were located beneath the oral mucosa of 

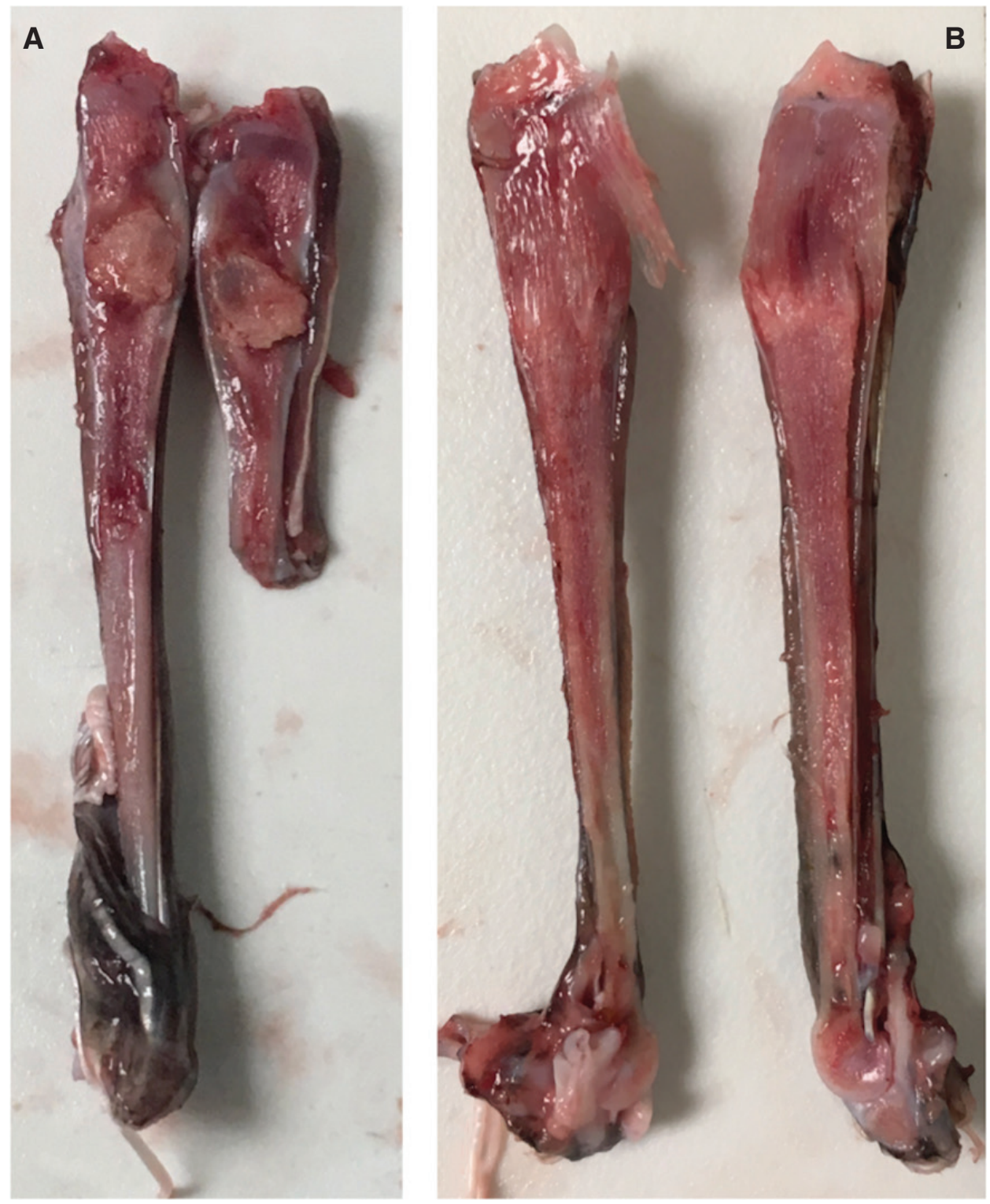

Fig. 2 Longitudinal sections through the tarsometatarsus of two SNEGs euthanized due to MBD. (a) Fracture with a callus or area of necrotic bone and (b) angular deformities and gelatinous-appearing soft bone at the proximal end of the tarsometatarsus.

$13 / 145(9 \%)$ birds. None of these parasites appeared to be directly related to the cause of death or euthanasia.

Eustrongylides spp. worms were present in the coelomic cavity of $43 / 145(30 \%)$ animals. They were found in all species examined and in all life stages examined except hatchling. Worms ranged in colour from red to black and were frequently observed in tubules adhered to the serosal surface of the ventriculus (Fig. 4a). In four cases, worms were tangled throughout the intestines and associated with a severe coelomitis that made it very difficult to separate the serosal surfaces of bowel loops (Fig. 4b).
In some severe cases, they extended into the body wall and liver and were associated with haemorrhage (Fig. $4 c-e)$. Of the animals with eustrongylidiasis, seven had liver abscesses, five had lung abscesses, three had severe airsacculitis and one had abscessation of a kidney lobe. Firm black adhesions to the ventriculus, believed to be resolving lesions, were found in nine animals (Fig. 4f).

Eustrongylidiasis was not believed to be a significant contributor to the cause of death or euthanasia in 21 infected animals due to relatively low parasite burdens, and/or because a different cause was readily apparent 


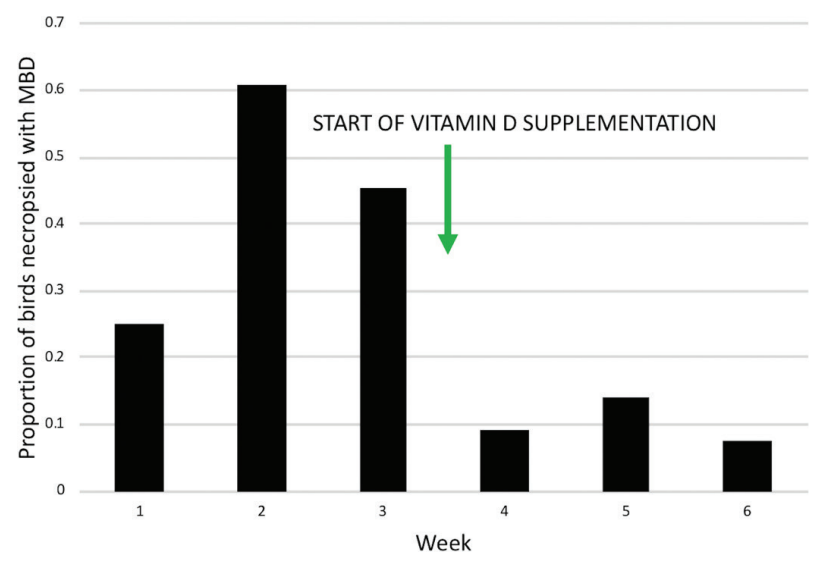

Fig. 3 Proportion of birds necropsied with signs of MBD by week during the six-week period that necropsies were performed, excluding cases that had signs of MBD at admission. Oral vitamin D supplementation began near the end of week three.

Table 3 Number of cases of MBD at intake relative to total admissions from 1 April to 9 August in 2017 and 2018.

\begin{tabular}{lcc}
\hline & MBD cases/total number of intakes \\
\hline Location & $\mathbf{2 0 1 7}$ & $\mathbf{2 0 1 8}$ \\
\hline Concord & $2 / 15(13 \%)$ & $0 / 20(0 \%)$ \\
Fairfield & $4 / 86(5 \%)$ & $3 / 39(8 \%)$ \\
Oakland & $2 / 105(2 \%)$ & $4 / 25(16 \%)$ \\
Sacramento & $5 / 36(14 \%)$ & $2 / 61(3 \%)$ \\
Santa Rosa & $11 / 258(4 \%)$ & $35 / 176(20 \%)$ \\
Total & $28 / 637(4 \%)$ & $51 / 446(11 \%)$ \\
\hline
\end{tabular}

(e.g., visceral gout or a history of euthanasia due to severe MBD). Based on the findings of high parasite burdens and associated lesions in internal organs, eustrongylidiasis was believed to be a contributing factor towards death in 10 animals, and a cause of hypothermia, anorexia and/ or weight loss leading to euthanasia in 12 animals.

\section{Visceral gout}

Of the 37 birds that died in care, 10 had visceral gout on necropsy, characterized grossly by white chalky deposits on the pericardium, liver and air sacs, and by pale kidneys on necropsy (Fig. 5). Species affected were SNEG, GREG, BCNH and GRHE, of which nine were fledglings and one was an older juvenile. At the time of death, eight were on a current course of the NSAID meloxicam at a dose of $0.5 \mathrm{mg} / \mathrm{kg}$ SID (once a day). This is on the lower end of the commonly used dosage range for birds; in many species, it is customary to use $1 \mathrm{mg} / \mathrm{kg}$ BID (twice a day) (Carpenter \& Marion 2018). The number of doses received before death ranged from 2 to 36 , with an average of 12 doses and a median of four doses. One bird died 13 days after receiving a single dose of meloxicam, and another died 31 days after receiving two doses. The association between meloxicam administration and visceral gout was significant $(p=0.0001)$. However, it is important to note that birds with gout did not represent the majority of birds treated with meloxicam; there were 57 necropsied birds that had received meloxicam (of which 10 had gout) and an additional 15 animals that had received meloxicam and were released during the study time period, with no clinical signs of gout.

\section{Intestinal perforation}

Intestinal perforation was observed in three $\mathrm{BCNH}$ and one GRHE and was characterized by gross spillage of intestinal contents into the coelomic cavity with resultant coelomitis and airsacculitis. Abscessation of the liver and/or lungs was found in three of these birds. In the most severe case, intestinal contents had spilled into thoracic airsacs (Fig. 6). "Gurgling" breathing sounds were noted by staff on this bird's record prior to euthanasia. Hypothermia, obtundation and regurgitation were the reason for euthanasia in two animals. The third animal died in care with visceral gout noted on necropsy, and the fourth was euthanized for an unrelated reason (hock luxation). All four birds were on a current course or had recently finished a course of meloxicam at a dose of $0.5 \mathrm{mg} / \mathrm{kg}$ SID (mean treatment length 17 days, range 9-31 days). The association between meloxicam administration and intestinal perforation was significant $(p=0.02)$.

\section{Joint infections}

Swelling in one or multiple joints was the cause of euthanasia for nine animals. Differentials included septic arthritis and trauma, although other signs of trauma such as bruising or wounds over the joints were not seen. When joint capsules were cut with a scalpel at necropsy, a semi-opaque white fluid was expressed. Cytology in two cases showed high numbers of polymorphonuclear leukocytes. Microorganisms were not observed.

\section{Rib fractures}

Over one-third of the animals necropsied had one or multiple rib fractures $(n=50)$, characterized by nodular rib enlargements that were present at variable points along the length of the rib (Fig. 7). Rib fractures did not appear to be significantly associated with MBD or other causes of death or euthanasia. 

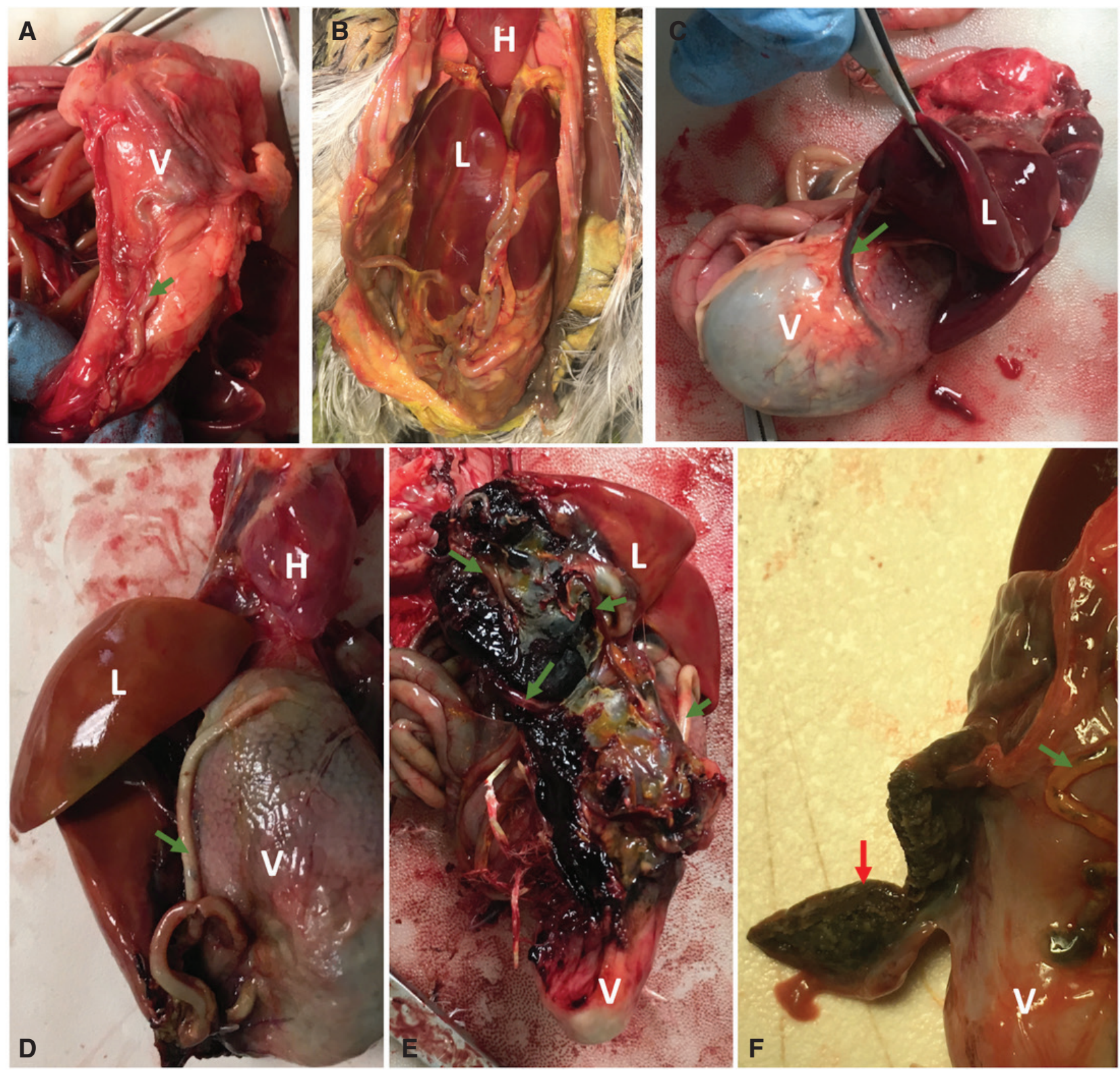

Fig. 4 (a) Eustrongylides spp. worm in a tubule adhered to the surface of the ventriculus of a GREG. (b) Eustrongylidiasis in a GRHE. There are numerous worms in the coelomic cavity entangled with the intestines and liver and associated with a severe coelomitis. (c) Eustrongylides worm extending from a tunnel on the ventriculus into the liver of a GRHE. (d) and (e) Eustrongylidiasis infection in a BCNH associated with extensive haemorrhage in the ventral body wall, liver and ventriculus. (f) Firm black plaque (red arrow) adhered to the ventriculus of a SNEG with eustrongylidiasis, believed to be a resolving lesion. It has been cross-sectioned with a scalpel. Green arrows = Eustrongylides worms. $\mathrm{H}=$ heart, $\mathrm{L}=$ liver, $\mathrm{V}=$ ventriculus.

\section{Other pathology}

There were 17 birds that died and 20 that were euthanized for declining clinically that did not have significant eustrongylidiasis, gout or intestinal perforation. Lung pathology was evident in 14 of these animals and included pale/brownish discoloration $(n=5)$, a dark/congested appearance $(n=7)$ and abscesses/granulomas $(n=2)$ (Fig. 8). Fungal plaques, presumed to be Aspergillus, were present in the thoracic air sacs of one fledgling GREG euthanized for dyspnoea. The liver was another common site of pathology. Findings (Table 4) included liver abscesses $(n=5)$, yellow/green discoloration of the liver $(n=6)$ 

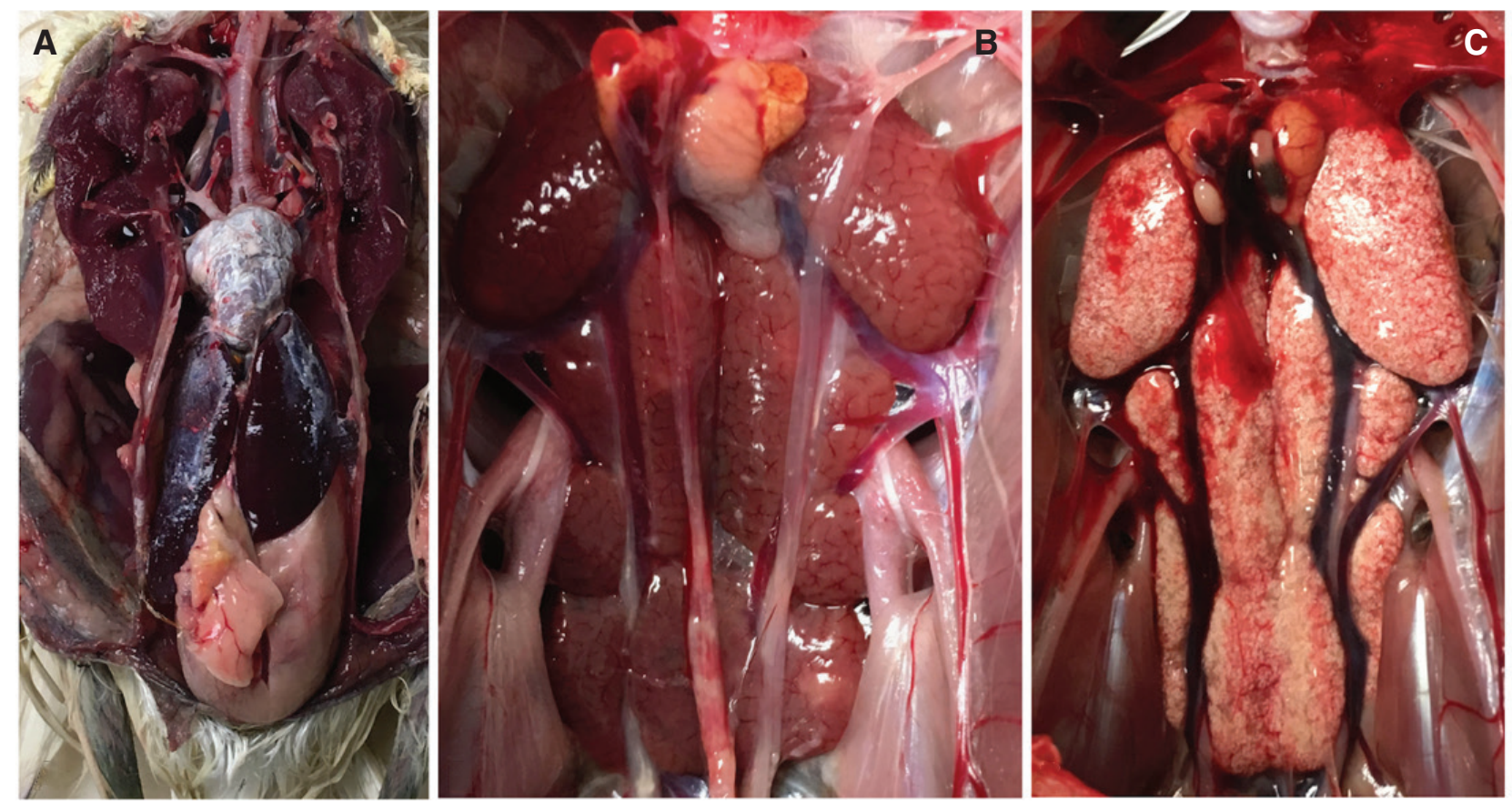

Fig. 5 (a) Visceral gout deposits on the pericardium and liver of a SNEG that died after 12 days of receiving the NSAID meloxicam at 0.5 mg/kg SID. (b) Normal appearing kidneys of a BCNH not treated with meloxicam and (c) kidneys of a SNEG that had visceral gout on necropsy that died after being treated with meloxicam for three days.

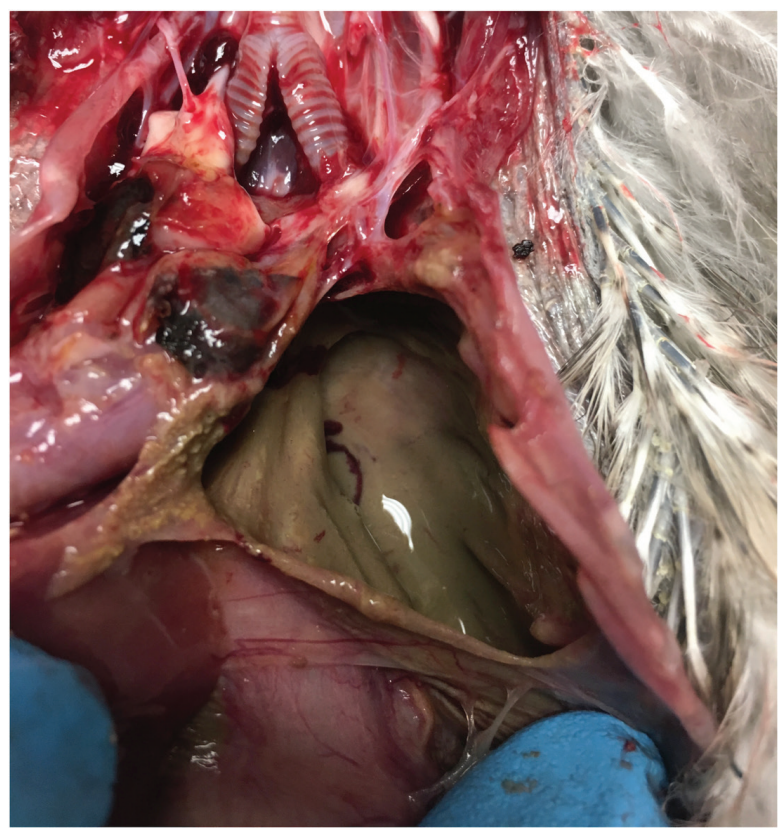

Fig. 6 Gross spillage of intestinal contents into the thoracic air sacs of a nestling BCNH that was euthanized for persistent hypothermia, regurgitation and obtundation after receiving meloxicam for nine days.

and hepatomegaly, defined as extension of the liver to the caudal body wall $(n=8)$. The majority $(n=5)$ of animals with hepatomegaly also had multifocal-coalescing $1 \mathrm{~mm}$ soft white nodules scattered throughout the liver parenchyma. Four birds had kidneys that were grossly pale relative to other tissues but with no evidence of visceral gout. Emaciation (BCS 0-2/9) was evident in 21 animals; further diagnostics were unavailable to determine the cause of death.

\section{Discussion}

MBD was the most common cause of euthanasia during the study time period. The aetiologies of MBD developing in care versus MBD present on intake were considered separately. Dietary imbalance was considered the likely cause of cases developing in care. During the study time period, birds were fed capelin (Mallotus villosus), a saltwater fish in the smelt family. In the previous year, they were fed night smelt (Spirinchus starksi), and there were very few cases of MBD developing in care. A low dietary calcium:phosphorus ratio is often looked to as the cause of MBD in captive birds and has been proposed as a cause of naturally occurring MBD in CAEG (Phalen et al. 2005). The birds in the current study were supplemented calcium carbonate for a dietary calcium:phosphorus ratio of 1.5:1 throughout the study period based on a nutritional analysis which found capelin to be low in calcium. For this reason, calcium deficiency was thought to be an unlikely cause of MBD, and vitamin D deficiency was considered. 

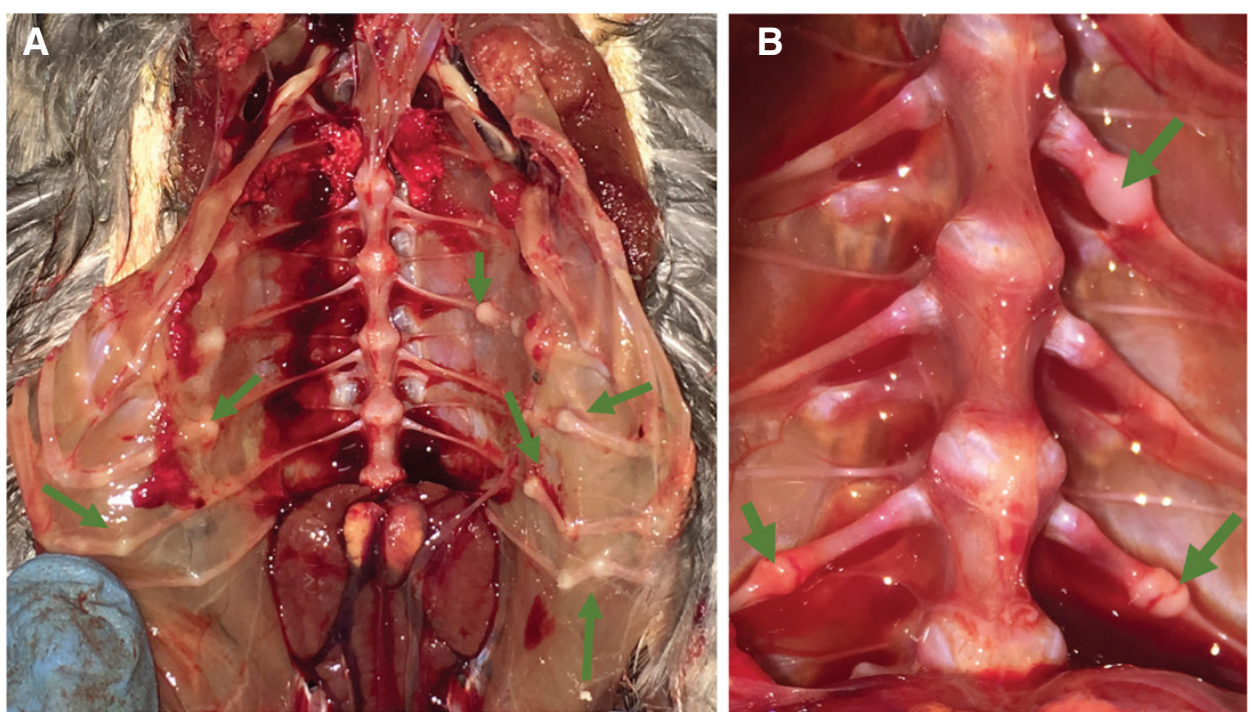

Fig. 7 (a) Multiple rib fractures (arrows) in a nestling GRHE that was euthanized for MBD. (b) Multiple rib fractures (arrows) in a nestling SNEG that was euthanized for septic arthritis.

In general, marine fish are believed to be a good source of vitamin D (Malesa-Ciecwierz \& Usydus 2015). However, there is a recent report of captive penguins developing low-serum vitamin D levels and MBD after being fed a diet of capelin (Hoopes \& Claus 2016), and nutritional analysis by another group found capelin to be relatively deficient in vitamin D, containing just $99 \mathrm{IU} / 100 \mathrm{~g}$ on a dry matter basis compared to $1257 \mathrm{IU} / 100 \mathrm{~g}$ in herring (Clupea spp.) (Slifka et al. 2001). The level of vitamin D measured in herring by Slifka et al. was set as the target for the supplementation at the rehabilitation centre in this study, and supplementation levels per bird were set based on estimates of daily fish ingestion. Capelin is often chosen as a feeder fish due to the availability and relative low cost compared to other fish with fewer nutritional shortcomings. Night smelt had become poorly available and prohibitively expensive by early 2018, hence the change in diet.

When oral vitamin $\mathrm{D}_{3}$ supplementation began, the number of MBD cases developing in care dropped dramatically, supporting the hypothesis that dietary vitamin $\mathrm{D}_{3}$ deficiency was the cause of the high number of cases developing in care. Vitamin D has a critical role in bone development; it is required for intestinal absorption of calcium and phosphorus, increases renal reabsorption of calcium and facilitates normal bone formation independent of its effect on calcium and phosphorus levels (De Matos 2008). The parathyroid gland enlargement observed in this study was likely due to stimulation in response to hypocalcaemia from inefficient absorption of dietary calcium from the gastrointestinal tract.
The positive response to vitamin D therapy in this study suggests that vitamin $\mathrm{D}_{3}$ deficiency should be considered as a possible cause of MBD in wildlife rehabilitation, and that growing piscivorous birds on a diet of capelin should be supplemented with vitamin $\mathrm{D}_{3}$. Routinely supplementing vitamin $\mathrm{D}_{3}$ to birds without a knowledge of how much their diet already contains is not recommended since the therapeutic index is relatively low (De Matos 2008), and toxicity is a risk with over-supplementation.

A search of records revealed there was also an increase in the number of cases with MBD on intake in the study year (2018) compared to the previous year (2017), attributable mostly to cases from Santa Rosa. Plasma biochemistry and toxicology may have been useful in determining the cause of this increase but were unavailable due to cost. It is possible that the wildfires in the Santa Rosa area in October 2017, at the time the most destructive in California's history, could have been a factor, either due to habitat destruction leading to a change in food availability or due to toxins released by combustion. A study of grey herons in the UK revealed an association between skeletal deformities and tissue levels of dioxins and dioxin-like compounds (Thompson et al. 2006). Other research has found a marked increase in environmental levels of dioxin-like compounds when homes are burned compared to forest or brush (Deardorff et al. 2008). The 2017 fires in Santa Rosa were remarkable for the number of manmade structures that were burned (Griggs et al. 2017); it is possible that this feature of the fires was responsible for releasing deformity-causing toxins into the 

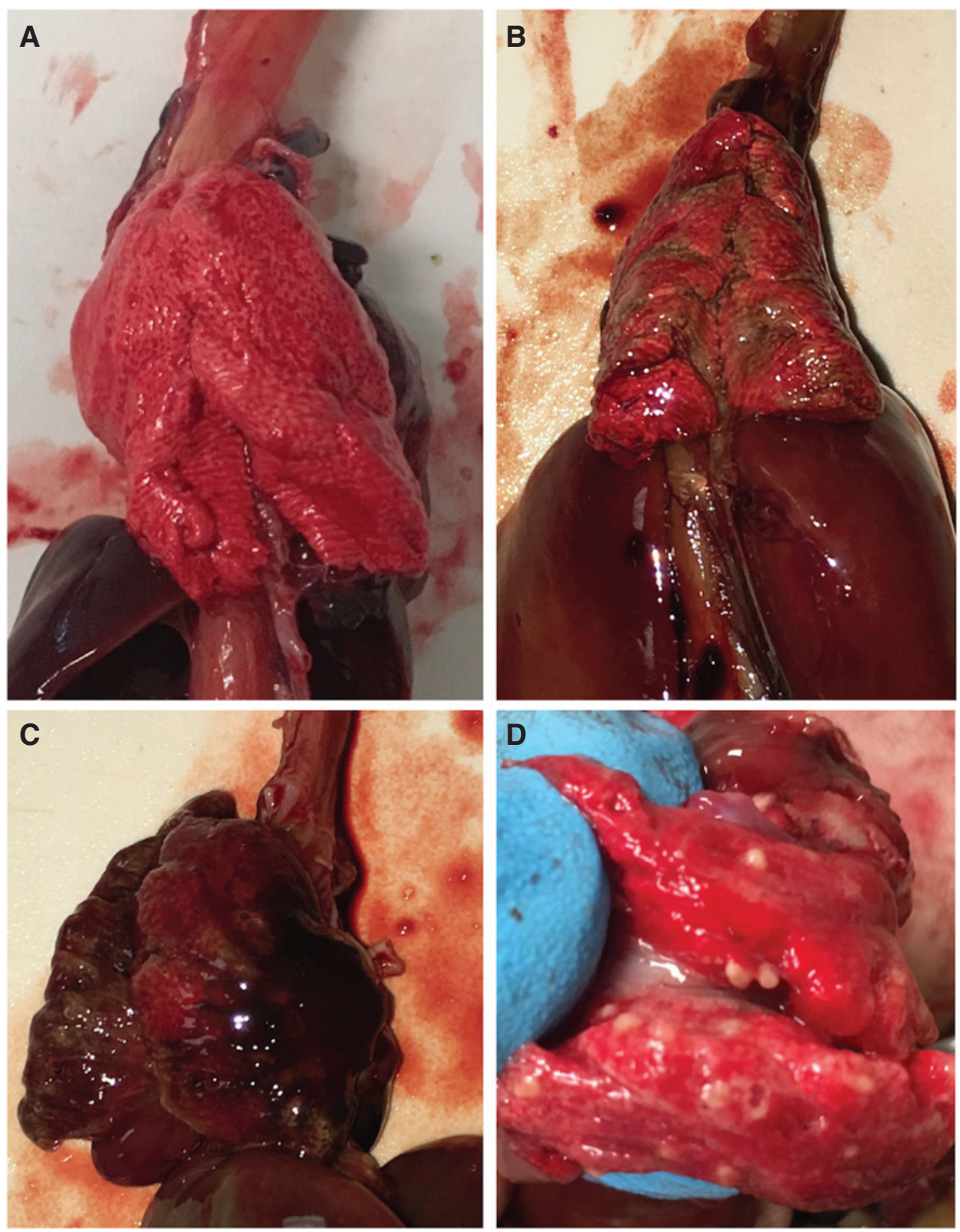

Fig. 8 (a) Normal-appearing lungs of a juvenile GREG. (b) Multifocal streaks of pale brown discoloration of the lungs of a fledgling SNEG that died after 21 days in care; necropsy was performed on the day of death. The only other pathology noted on necropsy was diffusely pale kidneys. (c) Congestion in the lungs of a fledgling SNEG that was euthanized after 19 days in care for obtundation, losing weight and regurgitation. Necropsy was performed on the day of death. A liver abscess was also noted on necropsy. (d) Granulomas in the lungs of a fledgling CAEG that was euthanized for hypothermia and weight loss after 26 days in care. No other gross pathology was noted on necropsy.

environment. Although the large heronry in Santa Rosa was not directly affected by fire, surrounding waterways where breeding herons and egrets are known to forage are downstream of the area burned (Madrone Audubon Society; Griggs et al. 2017). More studies are needed to determine the cause of MBD in young herons and egrets in this population.
The most common parasite observed on necropsy was Eustrongylides spp., which was present in $30 \%$ of cases and in all species examined. Prevalence was higher in this study population than previously reported in a study of eustrongylidiasis in SNEG and BCNH in northern California, where only $6 \%$ of nestlings collected from the wild were infected with the parasite (Franson \& Custer 1994). 
Table 4 Pathology present in animals that died or were euthanized for declining clinically that did not have an otherwise obvious cause of death.

\begin{tabular}{lc}
\hline Gross lesion & Number of animals \\
\hline Pale lungs & 5 \\
Congested lungs & 7 \\
Lung abscess/granuloma & 2 \\
Airsacculitis & 2 \\
Liver abscess & 5 \\
Hepatomegaly & 8 \\
Liver yellow/discoloured & 6 \\
Pale kidneys & 4 \\
Emaciated (0-2/9 BCS) & 21 \\
\hline
\end{tabular}

Possible explanations include an increase in prevalence of the parasite in California, a regional difference in prevalence within northern California or an overrepresentation of eustrongylidiasis in birds admitted to wildlife rehabilitation compared to the general wild population.

Infection with Eustrongylides spp. was a contributing factor towards death or a decision to euthanize in 22 animals. Of these, 10 animals died and 12 were euthanized for hypothermia, weakness and/or weight loss, which have been reported in heron nestlings experimentally infected with Eustrongylides spp. (Spalding \& Forrester 1993). Lesions in affected birds included the presence of worms in tortuous tunnels adhered to the ventriculus, extension of worms into the liver and body wall, abscessation of internal organs, and extensive peritonitis and airsacculitis, presumably due to translocation of gastrointestinal bacteria into the coelomic cavity through the break in the GI barrier caused by larval penetration through the ventricular wall, all of which have been reported in other studies on eustrongylidiasis in wading birds (Spalding \& Forrester 1993; Ziegler et al. 2000). Eustrongylidiasis has been associated with mortality rates of over $80 \%$ in nestling ardeids in some studies from the eastern United States (Wiese et al. 1977; Spalding et al. 1993). Mortality rates were not as high in this study, possibly due to a lower prevalence of the parasite in the region where this study took place, leading to lower parasite burdens and a higher proportion of non-lethal infections. It could also be due to differences in methodology-previous studies reporting high mortalities collected dead birds from their nests; these animals would not be seen by a wildlife rehabilitation centre as dead animals are not generally admitted. Eustrongylides should be suspected in young herons and egrets that die in rehabilitation or have persistent hypothermia, weakness, regurgitation and weight loss, even in cases where the parasite cannot be detected by abdominal palpation. Unfortunately, there are no effective treatments for eustrongylidiasis in birds. Even if worms are successfully killed, the bird may still be suffering from severe coelomitis and abscessation.

Other parasites noted on necropsy included nematodes of an unknown species in the ventriculus/proventriculus, trematodes in the oral cavity and oesophagus presumed to be Clinostomum marginatum and Avioserpens species in the mucosa of the oral cavity. These were believed to be incidental based on lack of apparent associated pathology and/or a definitive cause of death unrelated to the presence of the parasites. Every bird was routinely treated with antihelminthics (ivermectin and fenbendazole) on intake. Nematodes in the lumen of the ventriculus and proventriculus were noted only in birds that were euthanized or died within four days of intake, suggesting the treatment is effective against these parasites.

Visceral gout, an indicator of renal failure, and intestinal perforation were both significantly associated with treatment with meloxicam at $0.5 \mathrm{mg} / \mathrm{kg}$ SID. Meloxicam is a COX-2 preferential NSAID used for its analgesic properties (Engelhardt 1996). To the author's knowledge, there are no published reports of meloxicam causing mortality or clinically significant gastrointestinal- or nephron-toxicity in any avian species, despite it being a relatively well-researched drug in birds. One survey-based study of zoos, wildlife rehabilitation centres and exotic veterinarians found no recognized cases of mortality associated with meloxicam administration to 60 species in over 700 instances (Cuthbert et al. 2007). There have been studies examining changes in biochemical parameters and renal histology in African grey parrots (Montesinos et al. 2015), Japanese quail (Sinclair et al. 2012), Hispaniolan Amazon parrots (Dijkstra et al. 2015) and American kestrels (Summa et al. 2017). In all of these studies, birds were dosed every 12 hours, with doses ranging from 0.5 to $20 \mathrm{mg} / \mathrm{kg}$. None found a substantial alteration in clinical signs, renal biochemical parameters or renal histological lesions. Two of nine American kestrels dosed at $20 \mathrm{mg} / \mathrm{kg}$ BID for seven days had ulcers in the proventriculus and ventriculus detectable on histology but not grossly; this was not statistically significantly associated with meloxicam dose, though that may have been due to a small sample size (Summa et al. 2017).

Meloxicam has been reported to cause both nephrotoxicity and gastrointestinal perforation in mammals, although at recommended doses, these are rare side effects (Enberg et al. 2006; Dyer et al. 2010). Other NSAIDs are known to cause renal failure in avian species, perhaps most notably diclofenac in Gyps vultures (Oaks et al. 2004). One theory to explain the nephrotoxic effect of NSAIDs in birds is that decreased prostaglandin synthesis (due to COX enzyme inhibition) causes dysregulation of valves in the renal portal vein, leading to shunting of blood away from the kidneys (Meteyer et al. 2005). 
Another group proposed NSAID toxicity is the result of increased oxidative damage in renal tubular epithelial cells (Naidoo \& Swan 2009). NSAIDs are believed to cause intestinal ulceration, potentially leading to perforation, due to the interruption of prostaglandin-dependent mucosal defence mechanisms such as mucus and bicarbonate production and regulation of blood flow (Wallace 2001).

There are several reasons why the herons and egrets in this study may have been more susceptible to meloxicam toxicity than other studied avian species. They could be more sensitive to COX-2 inhibition or have differences in metabolic pathways, leading to a prolonged drug half-life, increasing the potential for toxicity. Although only one of the 10 birds with gout had an episode of hypothermia noted in its record, unrecorded hypothermia, dehydration or other illnesses may have predisposed some birds to kidney injury. All birds affected by renal and gastrointestinal toxicity in this study were juveniles, and it is possible that their young age could have predisposed them to toxicity. The association of visceral gout and intestinal perforation with meloxicam administration in this study suggests meloxicam should be used cautiously in herons and egrets and warrants further pharmacokinetic and toxicologic studies in these species.

Swollen joints were the cause of euthanasia in nine birds. Cytology of the joint fluid of two of these birds showed high numbers of polymorphonuclear leukocytes, consistent with inflammation either due to trauma or septic arthritis. No microorganisms were seen; however, it is possible to miss low numbers of infectious organisms on cytology. Culture could be performed to further try to distinguish these two differentials but was not performed here.

Trauma was the third leading cause of euthanasia after MBD and clinical decline, but is not discussed in further detail here as there is little to add from what was apparent ante-mortem. Rib fractures, which were not generally recognized ante-mortem, were highly prevalent in this study. They appeared to be incidental as there was no correlation with MBD or other causes of euthanasia or death. One plausible explanation is that ribs frequently fracture when young birds fall from their nests, especially in urban areas where they may fall onto concrete. As birds are required to move their body wall to breathe, it may be that these fractures take a long time to heal due to motion at fracture sites and are an unrecognized source of pain in these patients.

Birds without a clear cause of death or clinical decline frequently had pathology in the lungs and liver. Lung pathology included pale brown or dark red discoloration and pulmonary abscesses. Frequently, these were the only pathologies noted in these animals, suggesting they had a primary or at least contributing role in the death or clinical signs leading to euthanasia. However, caution should be taken when evaluating colour in the lungs on necropsy because of the propensity of this organ to develop post-mortem changes. One bird with congested-appearing lungs had a record of blood coming from its mouth prior to euthanasia, suggesting the lesion was ante-mortem, but other than that case, signs prior to death or euthanasia were non-specific for the respiratory system according to medical records.

Granulomas and abscesses in the lungs and liver suggest an infectious agent as a cause of illness in some birds. More work is needed to determine the identity of infectious organisms. Other pathology in the liver included discoloration, hepatomegaly and multifocal-coalescing $1 \mathrm{~mm}$ white nodules diffusely spread through the liver parenchyma. Possible ante-mortem aetiologies are numerous and include viral, parasitic or bacterial infections, neoplasia and metabolic disturbances. As with the lungs, care should be taken when interpreting liver colour as changes may occur post-mortem. More work is needed to determine the cause of liver and lung pathology in young herons and egrets.

The major limitation of this study was the reliance on gross pathology to determine a cause of death. A definitive diagnosis frequently requires additional diagnostic techniques such as microbiology, serology and histology. Gross evaluation of tissues such as the lungs and the gastrointestinal tract is complicated by the difficulty of distinguishing between ante- and post-mortem changes. That being said, in wildlife rehabilitation, more advanced diagnostics are often unavailable due to cost, and this study demonstrates that necropsy can be a useful tool in detecting both iatrogenic and natural causes of death and provides insight into the frustrating cases of animals that die suddenly or decline clinically despite medical and supportive cares. It is also important to state that the vast majority of animals in this study were young and admitted during a short period of time in one geographical area; it may not be appropriate to extrapolate findings to other populations of herons and egrets.

\section{Conclusions}

This study describes gross pathological findings in herons and egrets that died or were euthanized at a wildlife rehabilitation centre. Although it was not possible to determine a cause of death or clinical decline in every case, there are several important takeaways. Renal and/or gastrointestinal toxicity should be considered in an ardeid bird that suddenly dies or declines clinically during or after treatment with meloxicam. Vitamin D deficiency should be considered as a dietary cause of 
MBD in addition to calcium:phosphorus ratio imbalances. This study also supports the findings of previous work, showing that eustrongylidiasis is an important cause of mortality in young herons and egrets. More advanced diagnostics are needed to determine the significance and aetiology of other common lesions.

\section{Acknowledgments}

Thanks goes to the staff at International Bird Rescue for their care of these animals and assistance in saving carcasses for necropsy, as well as to Dr. Brian Murphy for his insight into the pathology of MBD.

\section{Disclosure statement}

The authors report no conflict of interest.

\section{Funding}

While no specific funding was designated for this project, International Bird Rescue provided facilities, materials, data, and personnel for this work.

\section{References}

Carpenter J.W. \& Marion C.J., editors. 2018. Carpenter's exotic animal formulary, $5^{\text {th }}$ edition. St. Louis, MO: Elsevier.

Coker S.M., Hernandez S.M., Kistler W.M., Curry S.E., Welch C.N., Barron H.W., Harsch S., Murray M.H. \& Yabsley M.J. 2017. Diversity and prevalence of hemoparasites of wading birds in Southern Florida, USA. International Journal for Parasitology: Parasites and Wildlife 6(3), 220-225. doi: 10.1016/j.ijppaw.2017.08.003.

Cuthbert R., Parry-Jones J., Green R.E. \& Pain D.J. 2007. NSAIDs and scavenging birds: potential impacts beyond Asia's critically endangered vultures. Biology Letters 3, 90-93. doi: 10.1098/rsbl.2006.0554.

Deardorff T.L., Karch N.J. \& Holm S.E. 2008. Dioxin levels in ash and soil generated in Southern California fires. Organohalogen Compounds 70, 2284-2288.

De Matos R. 2008. Calcium metabolism in birds. Veterinary Clinics of North America: Exotic Animal Practice 11, 59-82. doi: 10.1016/j.cvex.2007.09.005.

Dijkstra B., Guzman D.S., Gustavsen K., Owens S.D., Hass C., Kass P.H. \& Paul-Murphy J.R. 2015. Renal, gastrointestinal, and hemostatic effects of oral administration of meloxicam to Hispaniolan Amazon parrots (Amazona ventralis). American Journal of Veterinary Research 76(4), 308-317. doi: 10.2460/ajvr.76.4.308.

Dyer F., Diesel G., Cooles S. \& Tait A. 2010. Suspected adverse reactions, 2009. Veterinary Record 167, 118-121. doi: 10.1136/vr.c3650.

Enberg T.B., Braun L.D. \& Kuzma A.B. 2006. Gastrointestinal perforation in five dogs associated with the administration of meloxicam. Journal of Veterinary Emergency and Critical Care 16(1), 34-43.

Engelhardt G. 1996. Pharmacology of meloxicam, a new non-steroidal anti-inflammatory drug with an improved safety profile through preferential inhibition of COX-2. British Journal of Rheumatology 35(1), 4-12. doi: 10.1093/ rheumatology/35.suppl_1.4.

Franson C. \& Custer T.W. 1994. Eustrongylidiasis in wading birds from colonies in California, Texas, and Rhode Island, USA. Colonial Waterbirds 17(2), 168-172. doi: $10.2307 / 1521295$.

Griggs T., Lee J.C., Park H., Singhvi A., Wallace T., Ward J. \& Watkins D. 2017. Northern California fires have destroyed at least 5,700 buildings. The New York Times [Internet news service]. Accessed on the internet at https://www.nytimes. com/interactive/2017/10/14/us/california-fire-building-damage.html on 5 January 2019

Hoopes L. \& Clauss T. 2016. Investigation of a potential vitamin D3 deficiency in an African penguin (Spheniscus demersus) collection [abstract]. In International Association for Aquatic Medicine (IAAAM) Conference Proceedings 2016, May 21-26. Virginia Beach, VA. Accessed on the internet at https://www.vin.com/apputil/content/defaultadvl.aspx?pId=14818\&id=73122948 print=1 on 5 January 2019

Horgan M., Duerr R.S. \& Murphy B. 2021. Clinical and pathologic findings of an outbreak of vitamin D -responsive 3 metabolic bone disease in heron and egret (family ardeidae) chicks fed capelin (mallotus villosus). Journal of Zoo and Wildlife Medicine 51(4), 958-969. doi: 10.1638/2019-0238.

Madrone Audubon Society. West 9th Street Heronry [monograph on the internet]. Accessed on the internet at http://www.madroneaudubon.org/conservationwest-9th-street-heronry.php on 5 January 2019

Malesa-Ciecwierz M. \& Usydus Z. 2015. Vitamin D: can fish food-based solutions be used for reduction of vitamin D deficiency in Poland? Nutrition 31(1), 187-92. doi: 10.1016/j.nut.2014.07.003.

Meteyer C.U., Rideout B.A., Gilbert M., Shivaprasad H.L. \& Oaks J.L. 2005. Pathology and proposed pathophysiology of diclofenac poisoning in free-living and experimentally exposed oriental white-backed vultures (Gyps bengalensis). Journal of Wildlife Diseases 41(4), 707-716. doi: 10.7589/0090-3558-41.4.707.

Montesinos A., Ardiaca M., Juan-Sallés C. \& Tesouro M.A. 2015. Effects of meloxicam on hematologic and plasma biochemical analyte values and results of histologic examination of kidney biopsy specimens of African Grey Parrots (Psittacus erithacus). Journal of Avian Medicine and Surgery 29(1), 1-5. doi: 10.1647/2013-056.

Naidoo V. \& Swan G.E. 2009. Diclofenac toxicity in Gyps vulture is associated with decreased uric acid excretion and not renal portal vasoconstriction. Comparative Biochemistry and Physiology. Part C 149, 269-274. doi: 10.1016/j. cbpc.2008.07.014.

Oaks J.L., Gilbert M., Virani M.Z., Watson R.T., Meteyer C.U., Rideout B.A., Shivaprasad H.L., Ahmed S., Chaudhry M.J., Arshad M., Mahmood S., Ali A. \& Khan A.A. 2004. Diclofenac residues as the cause of vulture population 
decline in Pakistan. Nature 427, 630-633. doi: 10.1038/ nature02317.

Phalen D.N., Drew M.L., Contreras C., Roset K. \& Mora M. 2005. Naturally occurring secondary nutritional hyperparathyroidism in Cattle Egrets (Bubulcus ibis) from Central Texas. Journal of Wildlife Diseases 41, 401-415. doi: 10.7589/0090-3558-41.2.401.

Santos C.S., Brandão R., Monteiro M.S., Bastos A.C., Soares A.M. \& Loureiro S. 2017. Assessment of DNA damage in Ardea cinerea and Ciconia ciconia: a five-year study in Portuguese birds retrieved for rehabilitation. Ecotoxicology and Environmental Safety 136, 104-110. doi: 10.1016/j. ecoenv.2016.10.039.

Sinclair K.M., Church M.E., Farver T.B., Lowenstine L.J., Owens S.D. \& Paul-Murphy J. 2012. Effects of meloxicam on hematologic and plasma biochemical analysis variables and results of histologic examination of tissue specimens of Japanese Quail (Coturnix japonica). American Journal of Veterinary Research 73(11), 1720-1727. doi: 10.2460/ajvr.73.11.1720.

Slifka K., Crissey S.D., Khan S., Moser A., Chen T.C., Mathieu J. \& Holick M.F. 2001. Nutritional status in captive Bottlenose Dolphins (Tursiops truncates). In M. Edwards et al. (eds.): Proceedings of the fourth conference on zoo and wildlife nutrition. Pp. 151-157. Lake Buena Vista, FL: AZA Nutrition Advisory Group.

Spalding M.G., Bancroft G.T. \& Forrester D.J. 1993. The epizootiology of Eustrongylidosis in wading birds (Ciconiiformes) in Florida. Journal of Wildlife Diseases 29(2), 237-249. doi: 10.7589/0090-3558-29.2.237.
Spalding M.G. \& Forrester D.J. 1993. Pathogenesis of Eustrongylides ignotus (Nematoda: Dioctophymatoidea) in Ciconiiformes. Journal of Wildlife Diseases 29(2), 250-260. doi: 10.7589/0090-3558-29.2.250.

Summa N.M., Guzman D.S., Larrat S., Troncy E., Bird D.M., Lair S. \& Fitzgerald G. 2017. Evaluation of high dosages of oral meloxicam in American Kestrels (Falco sparverius). Journal of Avian Medicine and Surgery 31(2), 108-1 16. doi: 10.1647/2015-150.

Thompson H.M., Fernandes A., Rose M., White S. \& Blackburn A. 2006. Possible chemical causes of skeletal deformities in Grey Heron Nestlings (Ardea cinerea) in North Nottinghamshire, UK. Chemosphere 65, 400-409. doi: 10.1016/j.chemosphere.2006.02.007.

Van Franeker J.A. 2004. Save the North Sea Fulmarlitter-EcoQO manual part 1: collection and dissection procedures. Wageningen, Netherlands: Alterra. Accessed on the internet at http://edepot.wur.nl/40451 on 27 December 2018

Wallace J.L. 2001. Pathogenesis of NSAID-induced gastroduodenal mucosal injury. Best Practice $\theta$ Research Clinical Gastroenterology 15(5), 691-703. doi: 10.1053/ bega.2001.0229.

Wiese J.H., Davidson W.R. \& Nettles V.F. 1977. Large scale mortality of nestling ardeids caused by nematode infection. Journal of Wildlife Diseases 13(4), 376-382. doi: 10.7589/0090-3558-13.4.376.

Ziegler A.F., Welte S.C., Miller E.A. \& Nolan T.J. 2000. Eustrongylidiasis in eastern great blue herons (Ardea herodias). Avian Diseases 44, 443-448. doi: 10.2307/1592560. 